\title{
Philosophiques
}

\section{Jean-Luc Marion, De surcroît, PUF, Coll. « Perspective critique ", Paris, 2001, 208 pages.}

\section{Olivier Mathieu}

Volume 30, numéro 1, printemps 2003

Bernard Bolzano. Philosophie de la logique et théorie de la connaissance

URI : https://id.erudit.org/iderudit/007749ar

DOI : https://doi.org/10.7202/007749ar

Aller au sommaire du numéro

\section{Éditeur(s)}

Société de philosophie du Québec

ISSN

0316-2923 (imprimé)

1492-1391 (numérique)

Découvrir la revue

Citer ce compte rendu

Mathieu, O. (2003). Compte rendu de [Jean-Luc Marion, De surcroît, PUF, Coll. « Perspective critique », Paris, 2001, 208 pages.] Philosophiques, 30(1), 280-285. https://doi.org/10.7202/007749ar d'utilisation que vous pouvez consulter en ligne.

https://apropos.erudit.org/fr/usagers/politique-dutilisation/ 
l'attention du lecteur non plus sur la dite crise de l'historicité et de la spatialité en philosophie de l'histoire, comme thématique lancée au début du volume, mais sur l'intérêt général que peut présenter la géographie, en tant que mode de pensée, pour la pensée philosophique. La question n'est pas de se demander si et comment une épistémologie de la géographie doit être faite philosophiquement mais si la réflexion géographique peut apporter quelque principe utile à philosophie dans les champs de réflexion qui lui sont propres. Benoist aperçoit six «effets" possibles de la pensée géographique sur la pensée philosophique : 1) un «effet d'empiricité » qui consiste à présenter le monde dans sa matérialité, en tant que lieu que les hommes habitent, 2) un «effet de positivité » qui consiste à mettre de l'avant la description des choses telles qu'elles sont devant nous, par opposition à des démarches plus herméneutiques telle celle à laquelle a recours l'histoire, 3) un « effet de spatialisation» qui tient à la capacité de l'image de présenter quelque chose synthétiquement et dans la complexité des relations, 4) un «effet de politisation » qui voudrait dire que la géographie organise le monde en domaines politiquement constitués et introduit donc au sein de la définition des choses naturelles des rapports de pouvoirs, 5) un "effet critique " qui par ce découpage à la fois naturel et arbitraire du monde constitue une sorte de paradigme de la pensée catégorielle. L'espace géographique objectif constitué par la discipline ainsi que la spatialité de la représentation cartographique serait donc deux manières d'entendre l'espace ici en cause.

PIERRE LARUE

Université de Montréal

Jean-Luc Marion, De surcroît, PUF, Coll. «Perspective critique», Paris, 2001, 208 pages.

Avec De surcroît, Jean-Luc Marion vient clore le travail phénoménologique entamé près de 12 ans auparavant dans Réduction et donation; recherches sur Husserl, Heidegger et la phénoménologie (1989) où il n'était alors question que d'ouvrir la voie vers le principe de donation par l'entremise d'un «simple examen historique du développement de la méthode phénoménologique ${ }^{1}$ depuis la "percée husserlienne » de 1900-01. Faisant suite à Réduction et donation, Marion publiait en 1997 Étant donné, un essai téméraire où il tentait de reconduire la phénoménalité en son ensemble à la stricte donation, tâchant du même coup d'établir une frontière, mais non pas nécessairement une distance, entre le travail de la phénoménologie et celui de la métaphysique : la reconduction du phénomène à sa pure donation, hors des déterminations de l'objectité et de l'étance, avait pour effet immédiat de libérer la réflexion phénoménologique des apories d'une pensée métaphysique de la présence et, du même coup, de forcer la redéfinition du sujet, de l'ego, en fonction de sa position et de son rôle dans l'ordre de la donation - le sujet devint alors l'adonné. Toutefois, les insuffisances et les nombreuses carences d'Étant donné quant à l'explicitation de certaines thèses fondamentales, et particulièrement celle des phénomènes saturés, suscitèrent plus de questions que de réponses. Dès sa parution, donc, Étant donné exigeait déjà que l'on y revienne et que l'on en répète les acquis tout en les précisant. «De surcroît, donc.

1. Marion, J. L., Étant donné, Paris, PUF, collection «Épiméthée», 1997, p. 7 
Parce qu'il s'agit de l'excès du donné qui se montre. Parce qu'il s'agit aussi de l'exposer une nouvelle fois $»^{2}$.

Dernier volet d'une trilogie qui trouve ainsi son achèvement (?), De surcroît est l'occasion, ou les occasions, d'étudier et de mieux comprendre les phénomènes saturés en eux-mêmes, d'expliciter l'événement, l'idole, la chaire, et l'icône pour enfin atteindre à une pensée signifiante du phénomène saturé par excellence, la révélation. Nous disons : «les occasions»; en fait, De surcroît est la somme de différents textes, lesquels sont presque tous issus de conférences ou d'articles contemporains de, ou postérieurs à la rédaction d'Étant donné. Ainsi, le premier chapitre, «Phénoménologie de la donation et philosophie première ", fut-il publié pour une première fois en 1996, dans le $49^{\mathrm{e}}$ numéro de Philosophie, sous l'intitulé "L'autre philosophie première et la question de la donation »; la version publiée dans De surcroît en présente une version corrigée et remaniée et ne sert, en somme, qu'à introduire le lecteur au cœur de la problématique.

En effet, ce texte se borne essentiellement à reprendre, de manière récapitulative, les acquis fondamentaux de la phénoménologie de Marion depuis 1989 en les articulant autour du problème de la possibilité contemporaine d'une philosophie première. Ce problème, qui pour plusieurs peut sembler révolu, trouve son importance dans le cheminement de Marion en ce qu'il lui permet d'attester, à la fois historiquement et philosophiquement, du rôle fondamental et originaire qu'occupe la phénoménologie de la donation en philosophie. Historiquement d'abord, puisque c'est à l'aide des différentes occurrences d'une philosophie première dans l'histoire de la philosophie que Marion tente de penser la question de la primauté de la philosophie. D’Aristote à Kant, en passant par la philosophie médiévale, Marion identifie, et réfute, trois différentes versions de la prima philosophia selon qu'elles s'édifient sur la pensée de primats différents, soit l'ousia pour Aristote, la cause pour les médiévaux, et la noèse pour Kant et Descartes. Aucun de ces primats, conclut-il, n'est en mesure d'assurer la légitimité d'une quelconque philosophie première en ce qu'ils échouent tous à atteindre les vérités ultimes qu'ils visent pourtant : la substance est rapidement réduite à la présence, la cause première échappe invariablement à la causalité ellemême, et la noétique nous obstrue la voie vers un questionnement véritable du phénomène en son apparaître. Il faut donc penser, propose Marion, une autre voie, celle de la phénoménologie en tant que l'articulation philosophique du principe "Autant de réduction, autant de donation ». Ce principe, qui seul assure le primat de la donation et qui, conséquemment, permet d'échapper à la pensée métaphysique, offre une possibilité ultime pour la phénoménologie de prétendre au rôle de philosophie première. Toutefois, ce n'est qu'en dernière instance que toute phénoménalité se reconduit à la pure donation et qu'elle se dévoile ainsi. En effet, le domaine de la donation ne se découvrant qu'en dernier, c'est-à-dire au terme d'une réduction qui ne saurait admettre aucun principe a priori ni aucune transcendance, une réduction, donc, qui laisserait le phénomène apparaitre en son soi et depuis son soi sans lui imposer aucune détermination extérieure, il apparaît que le principe «Autant de réduction, autant de donation » est moins un principe premier qu'un principe dernier et ultime. Il faut donc en conclure, de dire Marion, que c'est en tant que philosophie dernière que la phénoménologie accède au statut de philosophie première.

Toutefois, cette primauté demeure fragile tant que n'est pas assuré le postulat sur lequel elle repose, à savoir que le phénomène possède toujours l'initiative de sa

2. De Surcroît, en quatrième de couverture 
manifestation, qu'il ne se montre que dans la stricte mesure où, toujours déjà, il s'est de lui-même donné. C'est à cette problématique, ouverte depuis le $7^{\mathrm{e}}$ chapitre de Sein und Zeit ${ }^{3}$, et déjà largement discutée tout au long du IIIIe livre d'Étant donné, que Marion occupe ses efforts d'analyse au $2^{\mathrm{e}}$ chapitre de De surcroît : "L'événement ou le phénomène advenant ». Issu d'une conférence donnée en 1999 à la Faculté de philosophie de l'Institut catholique de Paris, le texte de ce chapitre décline et explicite brièvement les déterminations fondamentales du phénomène saturé en général par l'entremise d'une analyse de l'événement. L'argumentation procède d'abord à une réduction du phénomène de l'événement qui le retire aux déterminations de l'objectité constituée et de la présence subsistante. Une fois établi que l'événement excède à chaque fois le cadre de ces catégories, il identifie le phénomène saturé à l'excès de l'intuition sur la signification (conceptuelle ou autre) et atteste du même coup que le phénomène saturé ne se montre que dans la mesure où il s'est donné de lui-même sans commune mesure. Sans quitter les sentiers ouverts par Étant donné, Marion poursuit et répertorie les phénomènes saturés selon les rubriques fournies par les catégories kantiennes, à savoir selon que l'excès d'intuition se produise par la quantité (événement), la qualité (idole), la relation (chair) ou la modalité (icône), puis précise les déterminations fondamentales de la donation du phénomène soit l'anamorphose, l'arrivage, la facticité, l'incidence et le caractère d'événementialité propre à tout phénomène saturé. Ces précisions permettent à Marion d'enchaîner avec une réflexion sur les différentes caractéristiques de l'adonné face à la manifestation du phénomène qui se donne : l'événementialité de sa temporalité, le phénomène de sa naissance, la place de l'ego dans la réduction au donné et sa résistance au révélé sont tour à tour évoqués puis brièvement analysés dans une langue beaucoup moins théorique que celle que l'on retrouve dans Étant donné.

À cet égard, on notera au passage que la langue employée dans De surcroît, ainsi que la généralité du propos, permettent au lecteur étranger aux textes de Marion de s'introduire sans peine au cœur même de sa réflexion. Du coup, ce texte s'offre à lui comme une clé de compréhension très utile pour la lecture de textes antérieurs plus particulièrement ceux nommés ci-haut. Par contre, le lecteur familier avec Étant donné et sa conceptualité notera quelques changements d'ordre lexical qui, s'ils ne provoquent pas de changements profonds dans la pensée de Marion, en altèrent néanmoins la dynamique. Au nombre de ces différences, on note la complète, et fort surprenante disparition du concept de "répons ${ }^{4}$, lequel joue un rôle clé dans Étant donné en ce qu'il permet l'explicitation de l'activité de l'adonné face à la manifestation du phénomène, et assure une continuité avec les thèses de Réduction et donation ${ }^{5}$. Marion emploie plutôt le concept de "résistance ", dont on sent bien qu'il doit renvoyer au même phénomène. Pourtant, ces concepts impliquent chacun une activité fort différente pour l'adonné qui les met en œuvre. Aussi, bien que l'on imagine sans trop de peine qu'il ne s'agisse là que d'une réponse ou d'un ajustement aux objections et

3. «Que le phénomène se définisse comme ce qui se montre en soi et à partir de soi, Heidegger l'a établi et fait admettre : "ce qui-se-montre-en-soi-même. " (Sein und Zeit, chap. 7, p. 31) Mais il a laissé largement indéterminée la façon dont peut se penser le soi à l'œuvre dans ce qui se montre. » Marion, De surcroît, p. 35

4. Cf. Étant donné, $\$ 28$

5. Voir le livre VI de Réduction et donation, plus particulièrement les $₫ 4$ sq. qui traitent de l'appel et de la revendication, ainsi que le livre V d'Étant donné, plus précisément les chapitres $\$ 26, \mathbb{2} 27, \mathbb{2 8}, \mathbb{2} 29$ qui s'attaquent à la problématique de l'articulation de l'appel et du répons. 
aux commentaires reçus depuis la parution d'Étant donné, il est regrettable, et fort dommageable pour la juste compréhension des thèses avancées, que les raisons de ces divergences demeurent inexpliquées : la distance lexicale entre les deux textes appelle de nombreux éclaircissements que De surcroît ne fait qu'effleurer, s'il ne les élude pas complètement. ${ }^{6}$

Le second chapitre, "L'idole ou l'éclat du tableau », marque également une certaine distance d'avec Étant donné à la seule différence, notable, que ce chapitre (inédit), ajoute de manière originale à la réflexion de 1997. Bien que certains développements restent très près du texte d'Étant donné - l'opposition entre voir et regarder, par exemple, qui calque à peu de choses près les pages 298-299 —, l'analyse phénoménologique du tableau - en l'occurrence, ceux de Rothko - offre une discussion pertinente et enrichissante de la visibilité propre à l'idole. Toutefois, le véritable apport de ce chapitre tient à ce qu'ouvre cette discussion, à savoir la possibilité d'une analyse de la distinction entre la face et la façade, et, du même coup, entre l'idole et l'icône. En accomplissant la reconduction de la visibilité du tableau au vu pur, c'est-à-dire à cette surface plane où rien ne se retire à la vue ou ne se laisse saisir et constituer par le regard - par le travail de présentation et d'apprésentation ${ }^{7}-$, Marion achève de dévoiler le phénomène saturé selon la qualité comme une façade, c'est-à-dire comme l'annulation de toute profondeur. Suivant en cela les analyses d'Emmanuel Lévinas dans Totalité et infini, Marion tire alors les conclusions éthiques qui s'imposent. En effet, dès lors qu'il est admis que l'idole réduit d'emblée ce qu'elle expose à un $v u$ pur et sans profondeur, il apparaît que tout le domaine de ce qui se retire à la vue, l'invisible, ne lui est alors accessible qu'au prix d'une terrible agression et d'une sauvage mutilation. Tel est précisément ce qui advient lorsque le tableau tente d'encadrer le visage d'autrui, la face. La figure d'autrui est phénoménolo-giquement comprise comme ce qui se manifeste et s'expose toujours déjà dans le «ni vu, ni connu » : l'adresse du regard de l'autre, ce regard qui m'envisage sans qu'il ne me soit jamais donné de le voir, la figure, en sa contre-intentionnalité irréductible, celle qui se donne depuis la noire profondeur de la pupille, voilà ce que l'idole détruit chaque fois qu'elle tente de mettre la face en façade; l'idole "tue» la face, la dé-visage. Du coup, cette compréhension donne à voir les limites et les normes, à la fois éthiques et esthétiques, de ce qu'il m'est permis de faire du visage d'autrui, et, a fortiori, d'autrui en général. C'est en cela que réside l'apport proprement original de De surcroît : l'exposition d'une normativité négative envers autrui — il s'agit à chaque fois d'un « ne pas", comme dans le "Tu ne tueras point " — à travers l'analyse du tableau, de l'idole. Bien qu'Étant donné laissait déjà entrevoir cette possibilité, rien ne laissait présager l'importance que Marion lui accorde ici tant l'emphase était alors mise sur la fonction radicalement individualisante du tableau. Du coup, ce n'est pas tant la distance lexicale, ni même théorique, qui exige des éclaircissements, mais plutôt l'originalité des thèses, lesquelles mériteraient d'être déployées et explicitées avec plus de précision.

Rien de tel avec le chapitre suivant, "La chair ou la donation du soi », dont le texte, rédigé à la même époque qu'Étant donné ${ }^{8}$, ne fait qu'élargir et préciser l'horizon

6. En fait, la disparition du répons est entièrement passée sous silence.

7. Cf. De Surcroît, p. 76; ou encore, Husserl, Les méditations cartésiennes, $\mathbb{S} 50$, Hua. 1, p.138 sq.

8. Conférence faite à Rome en janvier 97, puis publiée en 99 sous le titre «La prise de chaire comme donation du soi ", in "Incarnation, Archivio di Filosofia", éd. Marco M. Olivetti. 


\section{Philosophiques / Printemps 2003}

de la réflexion telle qu'elle se laissait déjà saisir en 97 ; il s'agit, à peu de chose près, de l'explicitation d'un déjà donné. L'exposition du phénomène saturé selon la relation débute par un retour critique sur les thèses de Descartes et Husserl touchant aux déterminations de la relation entre l'ego et ce qu'il convient de nommer, en un premier temps, son corps sentant. Faisant d'abord travailler Descartes contre lui-même, Marion tente de montrer comment l'assimilation du corps au monde extérieur en général — le «sentable» - ne résiste pas à l'intuition, implicite dans son œuvre, que le corps se démarque essentiellement des autres substances étendues par sa propre activité sensible, son "sentiment ». A fortiori, note Marion, la pensée de Descartes ${ }^{9}$ trahirait une antériorité du sentir, du corps sentant, à la cogitation : l'ego se donnerait d'abord comme corps sentant à travers une forme de sentir originaire. Il revient ensuite à Husserl de fournir les concepts nécessaires à la pensée d'une telle dichotomie entre le corps sentant et les corps extérieurs. C'est ici qu'intervient la distinction corps/chair (Körper/Leib) : la chair se donne comme ce qui me donne à mon corps; originaire et antérieure à toute forme de corporalité, elle fait apparaître le corps pour moi, m'y attache et m'y lie par le sentiment. Du coup, toute phénoménalisation du monde doit nécessairement passer par ma chair, laquelle assure toujours déjà le milieu et le moyen de son apparition pour moi : impossible de s'y soustraire! Voulant légitimer phénoménologiquement ce qu'il a dégagé des thèses de Descartes et Husserl, Marion passe ensuite en revue différents phénomènes (souffrance, plaisir et vieillissement) qui donnent à voir et à comprendre la relation de l'adonné à sa chair. Ceci lui permet enfin d'exposer la prise de chair comme l'accomplissement de l'individuation et de la facticité de l'adonné. C'est en effet ce qui découle du surcroît d'intuition qui accompagne la relation de l'adonné à ce qui se donne à lui : au-delà de la relation, «la chair ne donne au contraire [des autres phénomènes saturés] rien que l'ego lui-même, dans le temps même où tout donné se donne à lui » ${ }^{10}$. Cependant, cette caractérisation de l'ego semble poser problème lorsque intervient la nécessité de penser l'autre en son altérité irréductible. Comment, en effet, penser sa chair dès lors que seul son corps, substance étendue, se donne à moi : l'intuition qui me la donnerait ne consisterait, en somme, qu'en une analogie d'avec le rapport, senti et connu, de ma chair à mon corps. Du coup, la chair de l'autre serait réduite à la mienne, donc au même, et perdrait irrémédiablement son caractère d'altérité. De quelle manière puis-je penser l'autre sans réduire son altérité ?

Ce sera au chapitre suivant, "L'icône ou l'herméneutique sans fin ", de fournir la réponse à cette question. Ce texte, tiré d'une conférence donnée pour la première fois en 1998 dans un colloque de la "Deutsche Gesellschaft für philosophische Forschung », reprend, non sans une certaine redondance, les analyses faites plus tôt au chapitre 3 à propos du visible, de la façade et de la face. Toutefois, l'intérêt ne repose plus tant sur le pur vu, le parfaitement visible, que sur la visibilité de l'invisible. L'invisible, dans ce cas, c'est le visage de l'autre, ou plutôt son regard en tant même qu'il est posé sur moi et qu'il m'écrase sous le poids de son irréductible contre-intentionnalité. Or, ce regard, s'il ne m'est jamais donné de le voir, de le constituer, n'en est pas moins silencieusement "parlant ", voire même criant : me prêter à sa parole silencieuse exige que je me rende à lui, au double sens de m'y déplacer et de m'y résoudre. Le fait de l'irruption du visage d'autrui dans le visible, l'injonction de sa contre-intentionnalité, m'impose de

9. L'argument de Marion se fonde surtout sur les difficultés et les apories des deux premiers chapitres des Méditations.

10. De surcroît, p. 121 
m'assigner à lui, d'inverser, autant que faire se peut, les pôles de visibilité ${ }^{11}$. C'est à cette tâche indéfiniment répétée, puisque aucun concept ne saurait épuiser — et constituer - la signification de ce qui, d'emblée, déborde à chaque fois mon intentionnalité, qu'autrui me convoque : le visage de l'autre m'advient toujours avec l'exigence d'une herméneutique infinie.

Avec cette détermination de l'icône, Marion achève de décliner et d'expliciter les quatre types de phénomènes saturés qu'Étant donné laissait déjà entrevoir au chapitre 23 ; reste encore à explorer le phénomène saturé par excellence, à savoir la révélation. C'est ce que le dernier chapitre de l'essai entreprend sous l'intitulé «Au nom ou comment le taire ». Ce texte, issu d'une conférence contemporaine à la parution d'Étant donné, s'inscrit dans le cadre d'une discussion avec J. Derrida à propos du statut et de la légitimité de la "théologie négative». Contre Derrida et sa dénégation de la théologie négative, Marion tente de montrer comment cette forme de théologie se distingue de toute métaphysique de la présence ou d'onto-théo-logie, lesquelles réduiraient Dieu, et sa révélation, à l'horizon de l'être et de la présence, l'exposant ainsi aux apories propres à ces pensées. Pour parvenir à ses fins, Marion montrera comment cette théologie ouvre une troisième voie, au-delà des domaines du vrai et du faux, du bien et du mal, une voie qui ne laisse une seule possibilité au langage pour être dite : le silence. La disqualification du concept accomplit ce que Marion appelle la «dénomination ", laquelle aboutit finalement à une pragmatique théologique de l'absence : dé-nommer Dieu n'équivaut plus à le faire entrer dans l'ordre de la prédication mais, inversement, à nous inscrire dans l'orbe de son horizon (à travers le baptême, par exemple); l'usage du langage n'est plus théorique, il ne parle plus, mais pragmatique. C'est dans ce silence, dans cet aveu muet d'inconnaissance face à l'irréductible incompréhensibilité de Dieu, que le phénomène de la révélation peut préserver sa possibilité insigne, à savoir se donner dans une intuition qui déborde d'emblée tout accueil conceptuel.

OLIVIER MATHIEU

Université de Montréal

Engin F. Isin, Being Political. Genealogies of Citizenship, Minneapolis, University of Minnesota Press, collection «Political Science/Sociology », 2002, 335 pages.

Les généalogies de la citoyenneté d'Engin Isin ne sont pas simplement une histoire de la citoyenneté; il ne s'agit pas d'expliquer la naissance de la démocratie et les grandes dates de son évolution. L'objectif est plutôt de rendre compte des rapports de force déterminant cette histoire. La prémisse de départ de l'ouvrage est que l'histoire communément admise de la citoyenneté, loin d'être objective ou politiquement neutre, est le récit que nous ont offert les dominants - il emprunte l'expression à Pierre Bourdieu. Partant d'une représentation héroïque de la citoyenneté grecque antique, les dominants des époques subséquentes se seraient inscrits au cœur d'une tradition citoyenne

11. À cet égard, le phénomène saturé qu'est l'icône accomplit certainement mieux que tout autre l'opération phénoménologique de l'appel, thème capital tant dans Réduction et donation que dans Étant donné, et que De surcroît ne fait qu'évoquer brièvement en ces passages. 